\title{
Multiple Sclerosis and Trigeminal Neuralgia: A Well-Noted Association with Unknown Occurrence as a Presenting Symptom: Our Experience in Multi Centric Study
}

\author{
Luigi V Berra ${ }^{1}$, Daniele Armocida ${ }^{1 *}$, Andrea Di Rita ${ }^{2}$, Luca D Angelo ${ }^{1}$, Valerio Di Norcia ${ }^{1}$ and \\ Antonio Santoro ${ }^{1}$ \\ ${ }^{1}$ Department of Neurosurgery, Policlinico Umberto I, La Sapienza of Rome University, Italy \\ ${ }^{2}$ Department of Neurosurgery, Hospital San Carlo Borromeo, Milan, Italy
}

\begin{abstract}
Objective: The association between trigeminal neuralgia (TN) and multiple sclerosis (MS) is well established. In many cases series the frequency of TN is reported in patients with MS but information regarding the frequency of trigeminal pain as a symptom of disease onset is lacking. We present a multi centric analysis of SM-patients affected by TN where we investigated the onset of symptomatology and a current literature analysis.
\end{abstract}

Patients and Methods: We prospectively acquired clinical and radiological information of patients with MS and with TN that were visited at our clinics in two different departments. A pertinent literature review was carried out as well. We investigated the prevalence of TN in patients with MS and we noted whether facial pain was the symptom of MS onset.

Results: A total of 718 patients with multiple sclerosis were analyzed, of which 63 presented TN. The neuropathic facial pain was the presenting symptom of MS in 3 patients, that constituted $0.4 \%$ of cases. The results reported in the literature confirm a frequency of $0.4-1 \%$ of the cases in which the first symptom of MS is TN and are like those observed in our study.

Conclusions: Is important to investigate the rate of debut of TN in SM patients to set up the correct diagnostic and therapeutic program. Among the different types of neuropathic pain, TN in MS patients are a difficult to treat with a relevant impact on the quality of life. TN constitutes a clinical picture frequently associated with the MS. Nevertheless, $\mathrm{TN}$ as a clinical manifestation of the onset of MS is a rare occurrence and is just noted that is rarely the first symptom of disease.

KEYWORDS: Multiple sclerosis; Trigeminal Neuralgia; Facial Pain; Secondary Trigeminal Neuralgia

Quick Response Code:

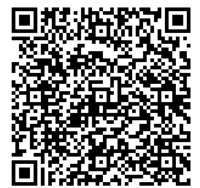

Address for correspondence: Daniele Armocida, Department of Neurosurgery, Policlinico Umberto I, La Sapienza of Rome University, Italy

Received: February 07, $2020 \quad$ Published: February 25, 2020

How to cite this article: LV Berra, D Armocida, DR Andrea, DA Luca, DN Valerio, S Antonio. Multiple Sclerosis and Trigeminal Neuralgia: A Well-Noted Association with Unknown Occurrence as a Presenting Symptom: Our Experience in Multi Centric Study. 2020 - 2(1) OAJBS. ID.000145. DOI: $10.38125 / O A J B S .000145$ 


\section{HIGHLIGHTS}

1. Multiple sclerosis is associated with different pain symptoms.

2. Trigeminal neuralgia is frequently associated to multiple sclerosis.

3. Trigeminal neuralgia as the first symptom of multiple sclerosis is a rare event.

\section{INTRODUCTION}

Multiple sclerosis (MS) is a chronic neuroinflammatory disease that affects the central nervous system, characterized by a variety of symptoms due to demyelination and axonal degeneration of the white matter of central nervous system. The reported prevalence of MS varies in different studies and countries and is reported between 60 and 200 cases per 100 [1]. Sensory disturbances and pain are common symptoms of MS [2,3]. Pains of any kind are symptoms frequently associated with MS. Among the various painful manifestations of MS, Trigeminal Neuralgia (TN) represents the most disabling and complex management neuropathic pain [4-7]. TN is a well-recognized cause of facial pain in the general population, although it is a relatively rare condition with a lifetime prevalence of up to $0.3 \%$ [8]. Secondary TN, both in the classical form and in the form of facial neuropathic pain, is an event that often accompanies this disease $[9,10]$. In many cases series the frequency of TN is reported in patients with MS but information regarding the frequency of trigeminal pain as a symptom of disease onset is lacking. In this study we aimed to investigate the incidence rate of TN as an onset symptom in a SM population and correlates these data with the current literature.

\section{PATIENTS AND METHODS}

We prospectively acquired clinical and radiological information of patients with MS and with TN that were visited at our clinics. We have analyzed the data acquired in a 10-year period, from January 2010 to December 2019, in two hospitals (Ospedale San Carlo Borromeo, Milan - Italy and Policlinico Umberto I - Sapienza, Rome - Italy), the prevalence of TN in patients with MS and we noted whether facial pain was the symptom of MS onset. The information acquired includes the presence of neuropathic facial pain and which was the symptom of onset of demyelinating disease. For all the included patients we recorded age, sex, location of pain, trigeminal root involved, clinical onset, time of diagnoses of SM. The diagnoses of SM was obtained in a specific neurological center following, radiological, clinical obtained by Macdonald criteria [11] It was agreed on that MS patients who had received a diagnosis of $\mathrm{TN}$ at any time, prior verification, during a routine clinical visit, of the following inclusion/exclusion criteria:

a) recurrent, unilateral, brief electric shock-like pain limited to the distribution of one or more divisions of the trigeminal nerve.

b) absence of constant pain.

c) Patients submitted at least a spinal -cranial MRI.

The above-said neurologists also examined clinical records and filled out a spreadsheet comprising selected patients' clinical data, information on prior and current medical treatments, and on type/timing of surgical procedures, if carried out. The following medications were considered "symptomatic" as they were used for acute pain relief: intravenous/oral steroids, opioids, paracetamol or NSAIDs; all other medical treatments (mainly antiepileptic drugs) were considered "preventive" as they were used for the long-term treatment of TN
All the patients included underwent a brain MRI scan included an high field 3 Tesla volumetric study with the following sequences: T2w, FLAIR, isotropic volumetric T1-weighted magnetizationprepared rapid acquisition gradient echo (MPRAGE) before and after intravenous administration of paramagnetic contrast agent; diffusion tensor sequences (DTI) completed our protocol for what concerns relationships between vascular and neural structures in the cerebellar -pontine angle's cistern.

The informed consent was approved by the Institutional Review Board of our Institution and Area Vasta Emilia Nord Ethics Committee approval was obtained for this study. Before medical treatment and surgical procedure, all the patients gave informed written explicit consent after appropriate information. Data reported in the study have been completely anonymized. No treatment randomization has been performed. This study is perfectly consistent with Helsinki declaration of Human Medical Research.

\section{RESULTS}

A total of 718 patients with MS where analyzed, of which 63 presented TN (8.8\%). Of the 63 patients with neuropathic facial pain, only in three of them this was the initial symptom. The most common presenting symptoms were paranesthesia (26\%) vertigo and dizziness (18\%) and ocular disturbances (16\%), followed by motor symptoms (14\%), dysarthria and tremor $(11 \%)$ and sphincter dysfunction (6\%) (Table 1$)$.

Table 1: Presenting symptoms of 718 patients with MS, A table show the characteristic onset of symptoms in our series.

\begin{tabular}{|c|c|}
\hline First Symptom of MS & Frequency (\%) \\
\hline Paraesthesia & $26 \%$ \\
\hline Vertigo and dizziness & $18 \%$ \\
\hline Ocular disturbances & $16 \%$ \\
\hline Motor symptoms & $14 \%$ \\
\hline Cerebellar (dysarthria and tremor) & $11 \%$ \\
\hline Sphincter dysfunction & $8 \%$ \\
\hline Others & $7 \%$ \\
\hline
\end{tabular}

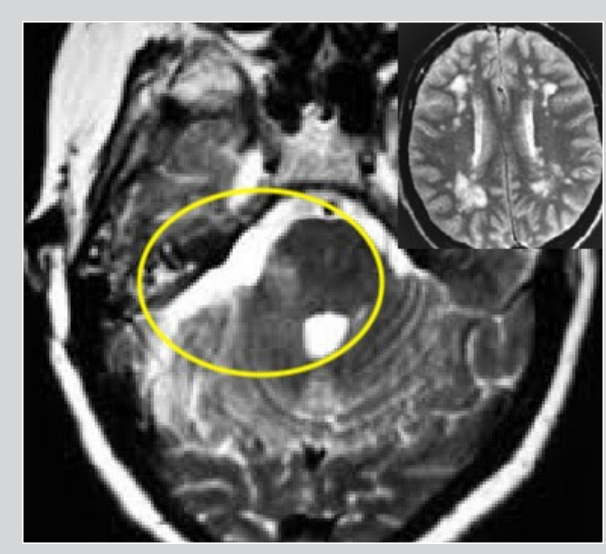

Figure 1: The image is an axial T2 weighted image on MRI illustrating typical vascular brainstem involvement, with a right involvement of the transverse pontine fibers near the trigeminal tract. The image on the right is an axial T2 weighted image of the sovratentorial space of an MS-patient, showing typical peripherally disseminated white matter lesions. 
Pain other than facial neuropathic pain was reported as a symptom of onset in 3\% of cases. Ultimately, in our experience, TN was the presenting symptom of MS only in 3 patients out of 718, which represents $0.4 \%$ of cases (Figure 1). The overall average age of onset was 41.3 years +/- SD $9.3 \mathrm{y}$, with a female to male ratio of 2.3 to 1 . There was no significant difference in the age of onset or average age between two group of MS patients with and without TN.

\section{DISCUSSION}

The association between SM and TN is well known [12,13], and the medical community has been aware of the co-occurrence of these two diseases for more than 100 years. Oppenheim has been credited as first describing the association, either in 1905 [14]. The incidence of $\mathrm{TN}$ in the general population, variably reported between studies, is into a range from 4.3 to 27 new cases per 100,000 people per year and a lifetime prevalence of 0.16 $0.3 \%$ in population-based studies. Patients with MS have a 20 -fold increased risk of developing TN [4]; TN is uncommon, but welldescribed phenomenon in patients with MS. Our series reports a prevalence of $8.8 \%$, but studies reported a rate between $1.9 \%$ and $4.9 \%$ of patients with MS to suffer from this neuropathic pain condition $[15,16]$, without differences between relapsing-remitting, secondary and primary progressive forms [15]; conversely MS is detected in $2 \%-14 \%$ of patients with TN [15], but it is still not well known in which number of cases the TN represents the onset symptom of MS. It just reported that TN in the majority appears after MS is up to 6\% [17] but up to $10 \%$ report TN as the first sign of MS and it can predate a second MS episode by up to 10 years [12]. In our collection of 718 evaluated patients, TN was the presenting symptom of MS only in $0.4 \%$ of cases. Just another single-center study conducted in British Columbia [15], Canada, reported that only five of 1182 individuals evaluated from 1980 to $1992 \mathrm{had}$ TN as the first presentation of MS, representing $0.4 \%$ of cases.

Foley et al. [18] reported that the overall prevalence of pain in MS is high, affecting more than $60 \%$ of individuals during their disease, without mentioning the onset of MS disease. Off the latter, a variable number is reported in the different studies of patients in which the pain is localized in the facial area [19-21]. [18] reported among 8590 SM patients' respondents to a survey, that the prevalence of TN was 830 (9.7\%). In the 588 participants with TN who reported the year that TN was diagnosed, TN preceded the diagnosis of MS in 88 (15.0\%). In one study in which both current and lifetime TN were reported, the lifetime prevalence of TN was $4.0 \%$, four-fold higher than the current prevalence $(1.2 \%)$ $[15,22]$. Most studies that have compared the age at diagnosis of TN in individuals with and without MS have found that TN occurs at younger ages in those with MS [23,24].

In practice, despite the article's title says that it is a common event, they report that about $1 \%$ of MS patients begin with TN [25]. The largest of these studies included participants from multiple centers but reported a low prevalence of TN of only $2 \%$. This may reflect the focus on current pain symptoms at the time the study was conducted rather than on lifetime diagnoses of TN, as reported in the present study [26]. We suppose these clinical data are useful for the choice of treatment; Although associated with a pathologically distinct disease process, MS-associated TN may be indistinguishable from classical TN. In these patients, the clinical presentation of TN is grossly like for the primary form, although more prolonged background pain and atypical features are reported $[9,24]$.
In MS patients with neuropathic facial pain, it may be unclear whether the pain is a sequela of MS plaques in the pons, which may affect trigeminal pathways but would not benefit from MVD, or of vascular compression in the $[5,12,27]$ prepontine cistern, which may benefit from surgical decompression. Collectively, the literature on treatment of TN in MS patients suggests that perhaps the pathophysiological mechanisms leading to their neuropathic facial pain cannot be simply dichotomized into patients with vascular compression and those with pontine plaques. [28-31]. A double insult mechanism for TN in MS patients was proposed by Truini et al. [32] after identifying co-occurrence of neurovascular compression and a demyelinating plaque to a greater degree ipsilateral vs. contralateral to facial pain in MS 31 patients with TN.

MS-related TN will often be very difficult to treat. Overall, up to $50 \%$ of patients with TN during first-line medical therapy (e.g., carbamazepine and oxcarbazepine) will experience a decline in the efficacy of these drugs in controlling their pain [33].

Rates of pain freedom in MS patients with TN have been reported in $20-74 \%$ of patients treated with [27,34,35] MVD, lower than for the general TN population. Given these concerns, some neurosurgeons will not offer MVD to patients with both TN and MS. However, withholding surgical treatment precludes offering potentially significant lasting relief to this vulnerable patient population [28]. In our study, we observed percentages like those previously reported in the literature of MS patients who have neuropathic facial pain as a manifestation of the disease's onset. We didn't find a significant difference in the age of onset of the two groups.

Nevertheless, our results and those available in the literature are quite homogeneous and lead us to affirm that the clinical onset of MS with TN represents a rather rare event, which occurs in 0.4$1 \%$ of cases. MS-related TN remains a challenge for neurologists and neurosurgeons owing to the lack of full comprehension of the complex pathogenesis at the basis of the onset of TN. PBC can be efficient in treating these patients [36].

\section{CONCLUSION}

Neuropathic pain is a common symptom in patients with MS. Is important to investigate the rate of debut of TN in SM patients to set up the correct diagnostic and therapeutic program. Among the different types of neuropathic pain, TN in MS patients are a difficult to treat with a relevant impact on the quality of life. Patients with MS experiencing TN find that daily life activities, work, mood, recreation and overall quality of life can be disrupted. TN constitutes a clinical picture frequently associated with the MS. Nevertheless, TN as a clinical manifestation of the onset of MS is a rare occurrence and is just noted that is rarely the first symptom of disease.

\section{REFERENCES}

1. Pugliatti M, Sotgiu S, Rosati G (2002) The worldwide prevalence of multiple sclerosis. Clin Neurol Neurosurg 104(3): 182-191.

2. Nurmikko TJ, Gupta S, Maclver K (2010) Multiple sclerosis-related central pain disorders. Curr Pain Headache Rep 14(3): 189-195.

3. Nick ST, Roberts C, Billiodeaux S, Davis DE, Zamanifekri B, et al. (2012) Multiple sclerosis and pain. Neurol Res 34(9): 829-841.

4. O Connor AB, Schwid SR, Herrmann DN, Markman JD, Dworkin RH (2008) Pain associated with multiple sclerosis: Systematic review and proposed classification. Pai 137(1): 96-111. 
5. Brisman R (2014) Treatment of trigeminal neuralgia associated with multiple sclerosis. World Neurosurg 81(3-4): 497-498.

6. Burkholder DB, Koehler PJ, Boes CJ (2017) Trigeminal Neuralgia and Multiple Sclerosis: A Historical Perspective. Can J Neurol Sci 44(5): 589593.

7. Ferraro D, Annovazzi P, Moccia M (2019) RIREMS (Rising Researchers in Multiple Sclerosis). characteristics and treatment of multiple Sclerosisrelated trigeminal neuralgia: An italian multi-center study. Mult Scler Relat Disord 19(37): 101461

8. Mueller D, Obermann M, Yoon MS (2011) Prevalence of trigeminal neuralgia and persistent idiopathic facial pain: a population-based study. Cephalalgia 31: 1542-1548.

9. Cruccu G, Biasiotta A, Di Rezze S, Fiorelli M, Galeotti F, et al. (2009) Trigeminal neuralgia and pain related to multiple sclerosis. Pain 143 186-191.

10. Di Stefano G, Maarbjerg S, Truini A (2019) Trigeminal neuralgia secondary to multiple sclerosis: from the clinical picture to the treatment options. J Headache Pain 20(1): 20

11. Fadda G, Brown RA, Longoni G, Castro DA, Mahony JO, et al. (2018) Canadian pediatric demyelinating disease network. MRI and laboratory features and the performance of international criteria in the diagnosis of multiple sclerosis in children and adolescents: A prospective cohort study. Lancet Child Adolesc Health 2(3): 191-204.

12. Zakrzewska JM, Wu J, Brathwaite TS (2018) A systematic review of the management of trigeminal neuralgia in patients with multiple sclerosis. World Neurosurg 111: 291-306.

13. Krishnan S, Bigder M, Kaufmann AM (2018) Long-term follow-up of multimodality treatment for multiple sclerosis-related trigeminal neuralgia. Acta Neurochir Wien 160(1): 135-144.

14. Marburg $O$ (1906) Die sogenannte akute multiple Sklerose (Encephalomyelitis periaxialis scleroticans). Jahrbücher für Psychiatrie und Neurologie 27: 213-312.

15. Hooge JP, Redekop WK (1995) Trigeminal neuralgia in multiple sclerosis. Neurology 45: 1294-1296.

16. Solaro C, Cella M, Signori A, Martinelli V, Radaelli M, et al. (2018) Neuropathic pain special interest group of the italian neurological society identifying neuropathic pain in patients with multiple sclerosis: A cross-sectional multicenter study using highly specific criteria. Neurol 265: 828-835.

17. Putzki N, Pfriem A, Limmroth V (2009) Prevalence of migraine, tensiontype headache and trigeminal neuralgia in multiple sclerosis. Eur J Neurol 16: 262-267.

18. Foley PL, Vesterinen HM, Laird BJ (2013) Prevalence and natura history of pain in adults with multiple sclerosis: Systematic review and metaanalysis. Pain 54: 632-642.

19. Lummel N, Mehrkens JH, Linn J, Buchholz G, Stahl R, et al. (2015) Diffusion tensor imaging of the trigeminal nerve in patients with trigeminal neuralgia due to multiple sclerosis. Neuroradiology 57(3): 259-267.
20. Tuleasca C, Carron R, Resseguier N, Donnet A, Roussel P, et al. (2014) Multiple sclerosis-related trigeminal neuralgia: A prospective series of 43 patients treated with gamma knife surgery with more than one year of follow-up. Stereotact Funct Neurosurg 92(4): 203-210.

21. Godazandeh K, Martinez SS, Wu J, Zakrzewska JM (2019) Trigeminal neuralgia: Comparison of characteristics and impact in patients with or without multiple sclerosis. Mult Scler Relat Disord 34: 41-46.

22. Martinelli BF, Colombo B, Annovazzi P (2008) Lifetime and actual prevalence of pain and headache in multiple sclerosis. Mult Scler 14: 514-521.

23. Jensen TS, Rasmussen P, Reske Nielsen E (1982) Association of trigeminal neuralgia with multiple sclerosis: Clinical and pathological features. Acta Neurol Scand 65: 182-189.

24. De Simone R, Marano E, Brescia MV (2005) A clinical comparison of trigeminal neuralgic pain in patients with and without underlying multiple sclerosis. Neurol Sci 26(2): S150-S151.

25. Fallata A, Salter A, Tyry T, Cutter GR, Marrie RA (2017) Trigeminal Neuralgia Commonly Precedes the Diagnosis of Multiple Sclerosis. Int J MS Care 19(5): 240-246.

26. Solaro C, Brichetto G, Amato MP (2004) The prevalence of pain in multiple sclerosis: A multicenter cross- sectional study. Neurology 63: 919-921.

27. Broggi G (2004) Operative findings and outcomes of microvascular decompression for trigeminal neuralgia in 35 patients affected by multiple sclerosis. Neurosurgery 55: 830-838; discussion 838-839.

28. Paulo DL, Lopez AM, Jermakowicz WJ, Yu H, Shah H, et al. (2020) Microvascular Decompression for Trigeminal Neuralgia in Patients with Multiple Sclerosis: Predictors of Treatment Success World Neurosurgery.

29. Olafson RA, Rushton JG, Sayre GP (1966) Trigeminal neuralgia in a patient with multiple sclerosis. An autopsy reports. J Neurosurg 24: 755-759.

30. Lazar ML, Kirkpatrick JB (1979) Trigeminal neuralgia and multiple sclerosis: demonstration of the plaque in an operative case. Neurosurgery 5: 711-717.

31. Ariai MS, Mallory GW, Pollock BE (2014) Outcomes after microvascular decompression for patients with trigeminal neuralgia and suspected multiple sclerosis. World Neurosurg 81: 599-603.

32. Truini A (2016) A dual concurrent mechanism explains trigeminal neuralgia in patients with multiple sclerosis. Neurology 86: 2094-2099.

33. Obermann M (2010) Treatment options in trigeminal neuralgia. Ther Adv Neurol Disord 3:107-115.

34. Crooks DA, Miles JB (1996) Trigeminal neuralgia due to vascular compression in multiple sclerosis--post-mortem findings. Br J Neurosurg 10: $85-88$

35. Gass A (1997) Trigeminal neuralgia in patients with multiple sclerosis: lesion localization with magnetic resonance imaging. Neurology 49: 1142-1144.

36. Solaro C, Trabucco E, Messmer UM (2013) Pain and multiple sclerosis: Pathophysiology and treatment. Curr Neurol Neurosci Rep 13: 320. 